\title{
Engineering $\mathrm{MoS}_{2}$ Nanosheets
} Anchored on Metal Organic Frameworks Derived Carbon Polyhedra for Superior Lithium and Potassium Storage

\author{
Binglong Rui ${ }^{1}$, Jiahui Li $^{1}$, Limin Chang ${ }^{1 *}$, Hairui Wang ${ }^{1,2}$, Li Lin $^{1}$, Yu Guo ${ }^{1}$ and Ping Nie ${ }^{1 *}$ \\ ${ }^{1}$ Key Laboratory of Preparation and Applications of Environmental Friendly Material, Ministry of Education, College of \\ Chemistry, Jilin Normal University, Changchun, China, ${ }^{2}$ School of Materials Science and Energy Engineering, Foshan \\ University, Foshan, China
}

OPEN ACCESS

Edited by:

Guiyin Xu,

Massachusetts Institute of Technology, United States

Reviewed by:

Zaiyuan Le,

A123 Systems, United States

Lifeng Chen,

National Institute of Advanced Industrial Science and Technology

(AIST), Japan

${ }^{*}$ Correspondence:

Limin Chang

aaaa2139@163.com

Ping Nie

pingnie@jInu.edu.cn

Specialty section:

This article was submitted to Electrochemical Energy Conversion and Storage,

a section of the journal

Frontiers in Energy Research

Received: 16 October 2019 Accepted: 18 November 2019 Published: 03 December 2019

Citation:

Rui B, Li J, Chang L, Wang H, Lin L, Guo $Y$ and Nie $P$ (2019) Engineering $\mathrm{MoS}_{2}$ Nanosheets Anchored on Metal Organic Frameworks Derived Carbon

Polyhedra for Superior Lithium and Potassium Storage. Front. Energy Res. 7:142 doi: 10.3389/fenrg.2019.00142
Metal organic frameworks (MOFs) have exhibited promising potential as a new platform for the preparation of porous functional materials for energy storage application. Herein, we report an approach to synthesize ultrathin $\mathrm{MoS}_{2}$ nanosheets decorated cobalt nanoparticles-containing porous carbon polyhedral with dense nitrogen doped carbon nanotubes (CNTs) backbone, where the ZIF-67 is used as the carbon and nitrogen sources for the growth of CNTs and the self-template for the carbon polyhedron. The composite shows a unique structure in which ultrathin $\mathrm{MoS}_{2}$ nanosheets are uniformly coated on the ZIF-67 derived carbon polyhedron. Owing to the synergistic effect from composition, morphology, and robust hollow structure composed of interconnected nitrogen doped CNTs networks and cobalt particles, the resulting ZIF-67-C@MoS 2 nanocomposites display high specific capacity of $568.5 \mathrm{mAh} \mathrm{g}^{-1}$ at a current density of $0.2 \mathrm{~A} \mathrm{~g}^{-1}$, superior rate capability (291.9 $\mathrm{mAh} \mathrm{g}^{-1}$ at $\left.5 \mathrm{~A} \mathrm{~g}^{-1}\right)$, and excellent cycling stability for over 500 cycles as lithium ion batteries anodes. The material also exhibits superior performance as new anodes for potassium ion batteries. The results provide a facile strategy for large-scale synthesis of high performance anode materials through low cost perspective and new insights for designing MOF-derived functional materials with unique structures for energy storage.

Keywords: metal-organic frameworks, $\mathrm{MoS}_{2}$, carbon polyhedra, lithium ion batteries, potassium ion batteries

\section{INTRODUCTION}

The need for large-scale energy storage on the smart grid and electric vehicles is growing rapidly. Electrochemical energy storage systems are highly desirable because they can offer high energy density, rate capability and rapid response (Dunn et al., 2011; Choi and Aurbach, 2016; Le et al., 2017; Liu et al., 2017, 2018; Peng et al., 2017; Sun et al., 2018; Davies et al., 2019; Ding et al., 2019). In the last two decades, lithium ion batteries (LIBs) have become increasingly popular because they possess superior energy density, light weight and long cycle life (Kovalenko et al., 2011; Wang et al., 2013; Li et al., 2016; Nie et al., 2017a, 2018; Griffith et al., 2018; Sun et al., 2019). The ever-growing demands for large scale applications of LIBs have opened a new area in advanced materials research. 
It is well-established that metal organic frameworks has gained enormous interests. As a new type of porous crystal material, it is composed of organic ligands and inorganic metal ions. Because of the high specific surface area, high porosity, and adjustable pore size, it widely used in many fields (Yunhong et al., 2017; Chen et al., 2018a; Mínguez Espallargas and Coronado, 2018) including catalysis (Chen et al., 2018c; Zhang et al., 2018c), hydrogen storage (Basdogan and Keskin, 2015; Kim et al., 2015), adsorption (Xue et al., 2013; Oveisi et al., 2018) and energy storage (Nie et al., 2014, 2015, 2017b; Wu and Lou, 2017; Xu et al., 2017; Wang et al., 2018). However, the conductivity of most MOFs is relatively low, and exhibiting low specific capacity as anode materials for lithium ion batteries (Chen et al., 2015). MOFs derived carbon materials could inherit the pristine porous structure of MOFs, and have tunable doping by heteroatoms and improved conductivity, thus providing a new platform for the synthesis of carbon based nanocomposites (Wang et al., 2017; Gadipelli et al., 2019). Jagadeesh et al. fabricated a graphitic carbon-coated cobalt for amination catalysts through pyrolysis of Co-MOFs precursors. The well-developed Co catalyst enables the effective synthesis of amines, amino acid derivatives, and more complex drug targets (Chen and $\mathrm{Xu}, 2017$; Jagadeesh et al., 2017). Three-dimensional (3D) carbon nanomaterials have attracted considerable attention as promising electrodes for electrochemical energy storage application due to their unique structural characteristics of interlinked architecture (Chen et al., 2017a). 3D porous MnO/C-N nanocomposites have been prepared by using renewable rapeseed pollen via a facile immersion-annealing route (Chen et al., 2017c), which showed excellent electrochemical performance in terms of high specific capacity, long cycling life over 400 cycles and super rate capability. Chen et al. reported the synthesis of hollow particle-based nitrogen-doped carbon nanofibers by embedding ultrafine zeolitic imidazolate framework (ZIF-8) nanoparticles into electrospun polyacrylonitrile fibers followed by carbonization (Chen et al., 2017b). When used as electrodes for supercapacitors, the hierarchical porous nanofibers exhibits superior electrochemical performance with remarkable specific capacitances, high energy/power density as well as outstanding cycling stability for 10,000 cycles at $5 \mathrm{~A} \mathrm{~g}^{-1}$.

Since the successful preparation of single layer graphene in 2004 (Novoselov et al., 2004), two-dimensional (2D) materials including transition metal dichalcogenides (TMDs), metal coordination polymer, layered transition metal oxide, have been extensively explored for energy storage application owing to outstanding mechanical flexibility and planar geometry (Zhang et al., 2018b). Among 2D transition metal dichalcogenides, $\mathrm{MoS}_{2}$ facilitates the insertion and extraction of alkali ions because of its weak inter-layer van der Waals force and a high theoretical specific capacity due to its multi-electron transfer mechanism (Tian et al., 2019). In addition, the material has the merit of low cost, thus has received considerable interests as promising anode materials for alkali-ion batteries (Jiao et al., 2017; Xie et al., 2017; Xu et al., 2018; Jiang et al., 2019). However, $\mathrm{MoS}_{2}$ suffers from the issues of fast capacity decay caused by mechanical strain induced during charge/discharge process and low rate capability because of intrinsically poor conductivity. When used as a host for lithium storage, besides intercalation, $\mathrm{MoS}_{2}$ can be converted into Mo particles and $\mathrm{Li}_{2} \mathrm{~S}$ with additional substantial capacity and a 103\% volume expansion (Xiao et al., 2010; Stephenson et al., 2014; Zhu et al., 2014). Li intercalation changes the Mo coordination from trigonal-prismatic to octahedral coordination. Furthermore, the poor electronic/ionic conductivity between two adjacent S-Mo-S layers also limits their applications.

Inspired by the above considerations, we herein report a strategy to synthesize ultrathin $\mathrm{MoS}_{2}$ decorated cobalt nanoparticles-containing porous carbon polyhedral with dense carbon nanotubes backbone, where the ZIF-67 is used as the carbon and nitrogen sources for the growth of carbon nanotubes and the self-template for the polyhedron structure. The material shows a unique structure in which ultrathin $\mathrm{MoS}_{2}$ nanosheets are uniformly coated on the ZIF-67 derived carbon polyhedron. With many advantageous features in composition, morphology, and robust hollow structure with nitrogen doped CNTs, the resulting ZIF-67-C@MoS 2 nanocomposite displays high specific capacity of $568.5 \mathrm{mAh} \mathrm{g}^{-1}$ at $0.2 \mathrm{~A} \mathrm{~g}^{-1}$, superior rate capability and excellent cycling stability $\left(350.4 \mathrm{mAh} \mathrm{g}^{-1}\right.$ upon 500 cycles) as lithium ion batteries anodes. It also exhibited superior performance as new anodes for potassium ion batteries.

\section{EXPERIMENTAL}

\section{Materials Synthesis Synthesis of ZIF-67-C}

ZIF-67-derived carbon materials were synthesized as follows. Firstly, 2-methylimidazole $(1.97 \mathrm{~g})$ and $\mathrm{Co}\left(\mathrm{NO}_{3}\right)_{2} \cdot 6 \mathrm{H}_{2} \mathrm{O}$ $(1.746 \mathrm{~g})$ was dissolved in a mixed solution of $20 \mathrm{~mL}$ methanol and $20 \mathrm{~mL}$ ethanol, respectively. The above two solutions were then mixed together under continuous stirring, and the final solution was aged at room temperature for $24 \mathrm{~h}$. After that, the purple precipitate was collected by centrifugation, washed in ethanol several times and dried at $60^{\circ} \mathrm{C}$. For the synthesis of the ZIF-67 derived carbon, the ZIF-67 particles were heated to $350^{\circ} \mathrm{C}$ for $1.5 \mathrm{~h}$, then $800^{\circ} \mathrm{C}$ for $3.5 \mathrm{~h}$ with a ramp rate of $2^{\circ} \mathrm{C}$ $\min ^{-1}$ under $\mathrm{Ar} / \mathrm{H}_{2}$ atmosphere (9:1 in vol. ratio). After cooling down to room temperature, the carbonized black powders were immersed in $0.5 \mathrm{M} \mathrm{H}_{2} \mathrm{SO}_{4}$ solution for $6 \mathrm{~h}$. The resulting ZIF-67 derived carbon was collected by centrifugation, washed with DI water, then dried at $60^{\circ} \mathrm{C}$, the resulting carbon was denoted as ZIF-67-C.

\section{Synthesis of ZIF-67-C@MoS 2}

The as-prepared ZIF-67 (30 mg) was added into $25 \mathrm{~mL} \mathrm{C}_{6} \mathrm{H}_{12} \mathrm{O}_{6}$ solution $(0.248 \mathrm{~g})$ and ultrasonication for several minutes. Then, $0.3 \mathrm{~g} \mathrm{Na}_{2} \mathrm{MoO}_{4} \cdot 2 \mathrm{H}_{2} \mathrm{O}$ and $0.6 \mathrm{~g} \mathrm{CH}_{4} \mathrm{~N}_{2} \mathrm{~S}$ were in order added into above solution. After stirring for $1 \mathrm{~h}$, the dispersion was transferred into a $50 \mathrm{~mL}$ Teflon-lined autoclave and heated at $200^{\circ} \mathrm{C}$ for $24 \mathrm{~h}$. The as prepared black powder was washed with deionized water and then dried at vacuum overnight. Finally, the samples were annealed at $500^{\circ} \mathrm{C}$ for $4 \mathrm{~h}$ in an argon flow with a heating rate of $1^{\circ} \mathrm{C} \min ^{-1}$. The obtained sample was marked as ZIF-67-C@MoS 2 . 


\section{Characterization}

Powder X-ray diffraction (XRD) (Rigaku d/max PC2500) with $\mathrm{Cu} \mathrm{K} \alpha$ radiation was used to determine the crystal structure of the products. The morphology and microstructure of the samples were observed using field-emission scanning electron microscopy (FESEM, JSM-7800F).

\section{Electrochemical Measurements}

The electrochemical performances were measured by using CR2032 type coin cell. The working electrodes were prepared by slurry coating procedure. The working electrode was prepared by mixing active material, acetylene black and carboxymethyl cellulose in a weight ratio of 80:10:10 in water. Then the slurry was spread onto an copper foil and dried under vacuum at $70^{\circ} \mathrm{C}$ for $12 \mathrm{~h}$. The cell assembly was performed in an argonfilled glove box. For lithium ion batteries, the electrolyte used was $1 \mathrm{M} \mathrm{LiPF} 6$ that was dissolved in a mixture of ethylene carbonate (EC) and dimethyl carbonate (DMC) (1:1 v/v) with 5\% fluoroethylene carbonate (FEC). Pure lithium metal was used as counter electrode and a polypropylene membrane as separator. For potassium ion batteries, the electrolyte was $0.8 \mathrm{M} \mathrm{KPF} 6$ dissolved in a mixed solution of ethylene carbonate (EC) and diethyl carbonate (DEC) (1:1, vol\%). Potassium metal was used as counter electrode, glass microfiber membrane as separators, respectively. Galvanostatic measurements were performed under different current densities between 0.01 and $3.0 \mathrm{~V}$ using a CT2001A cell test instrument (LAND Electronic Co.). Cyclic voltammetry $(\mathrm{CV})$ was measured using a electrochemical workstation in the voltage range of $0.01-3.0 \mathrm{~V}$.

\section{RESULTS AND DISCUSSION}

We designed and synthesized ultrathin $\mathrm{MoS}_{2}$ rooted on cobalt nanoparticles-containing porous carbon polyhedral with dense

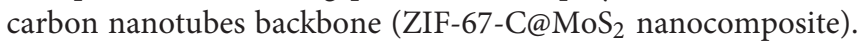
The fabrication process for the ZIF-67-C@MoS 2 composite is shown in Figure 1. During the preparation process, the ZIF67 serves not only as the carbon and nitrogen sources for the growth of CNTs, but also as the self-template for the polyhedron structure. The obtained ZIF-67-C retained a welldefined polyhedral morphology with dense CNTs rooted from both the inner and outer surface of the polyhedron. Then ultrathin $\mathrm{MoS}_{2}$ covered uniformly on the surface of the ZIF67-C polyhedron via a facile hydrothermal approach. The insitu grown CNTs and cobalt particles could form an integrated conductive network, which could express the issues of $\mathrm{MoS}_{2}$ anode including electronic conductivity and volume expansion upon cycling. Furthermore, the robust hollow structure ensures an efficient interfacial contact between active material and electrolyte, beneficial for fast charge-discharge properties.

The morphologies and structures of the as prepared ZIF-67, ZIF-67-C, and ZIF-67-C@ $\mathrm{MoS}_{2}$ composites were examined by field emission scanning electron microscopy (FE-SEM). From the SEM images of ZIF-67 (Figures 2a,b), it can be seen that ZIF-67 possesses a well-defined polyhedral morphology with a particle size of $c a .1-1.5 \mu \mathrm{m}$, and each rhombic dodecahedron has a smooth surface, consistent with previous reports in literature (Jiang et al., 2013). As shown in Figures 2c,d of the SEM images of ZIF-67-C, the rhombic dodecahedron and size are well-retained after carbonization, and the surface becomes

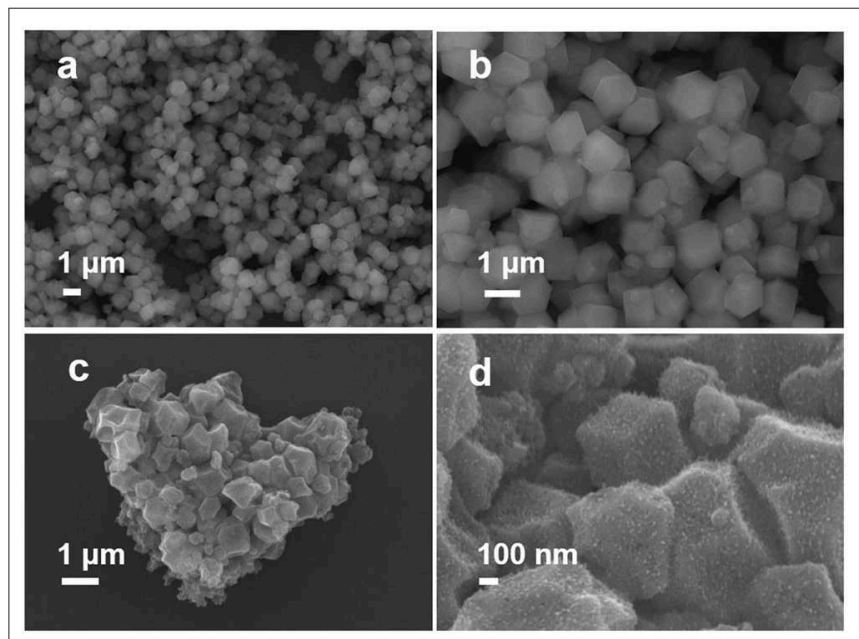

FIGURE 2 | SEM images of ZIF-67 (a,b), ZIF-67-C (c,d).

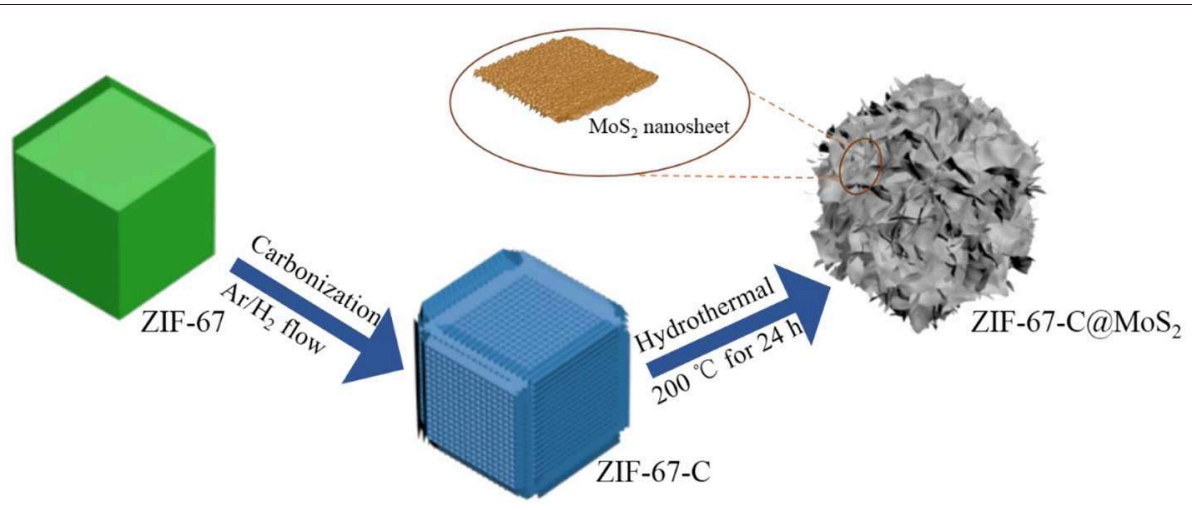

FIGURE 1 | Schematic illustration for the synthesis of ZIF-67-C@MoS 2 nanocomposite. 


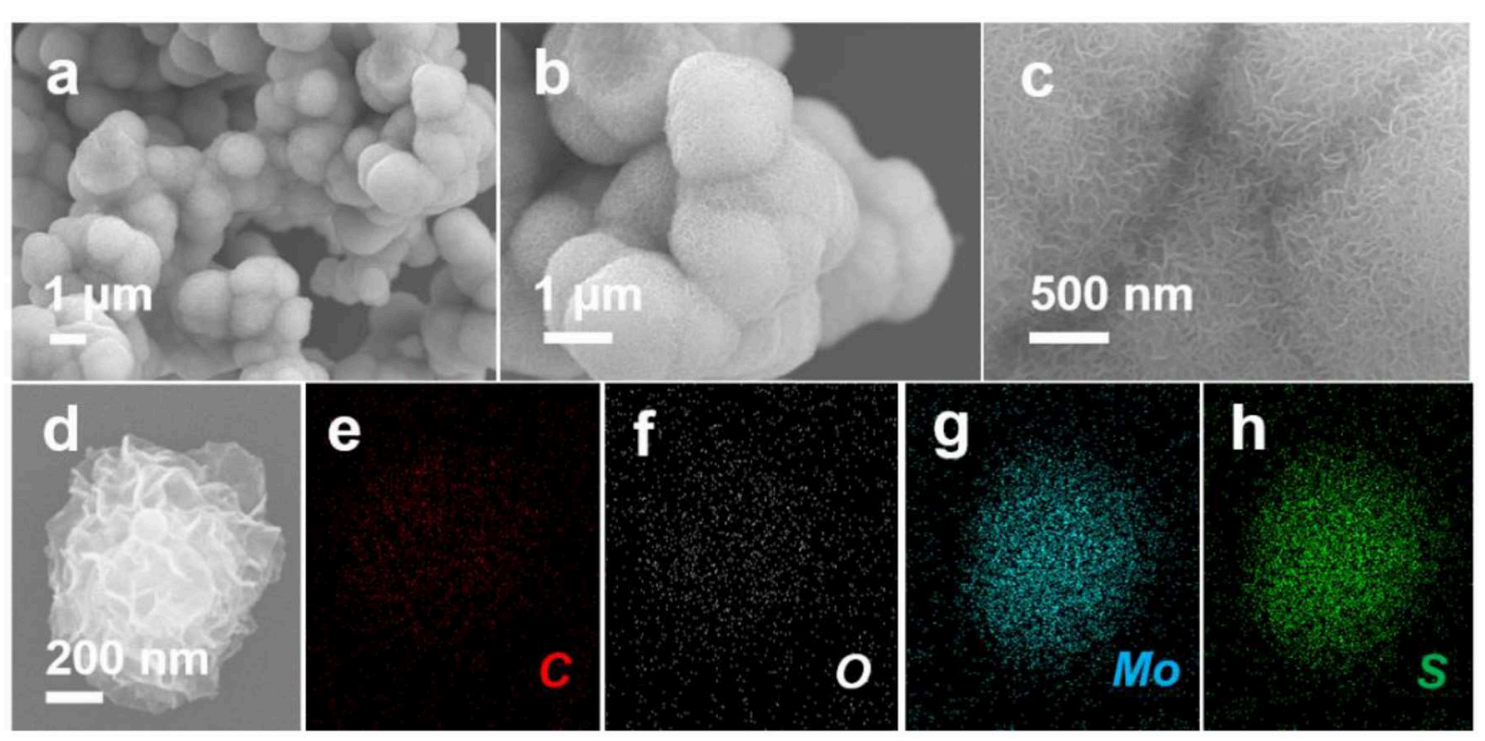

FIGURE 3 | SEM images of ZIF-67-C@MoS 2 (a-c), elemental mapping showing the uniform distribution of C, O, Mo, and S elements in ZIF-67-C@MoS 2 composite (d-h).

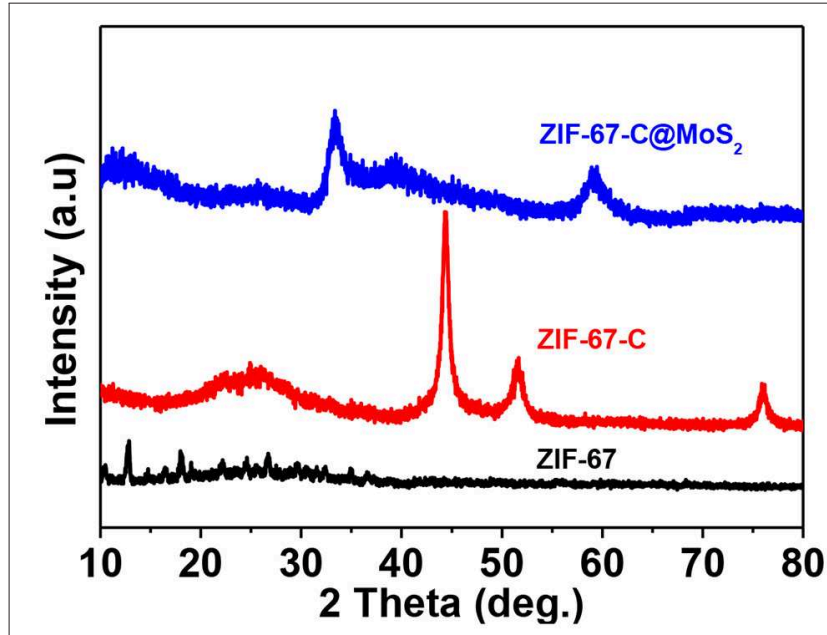

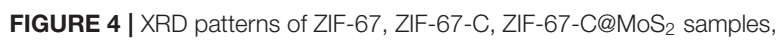
respectively.

rough and many carbon nanotubes covered can be observed. Furthermore, the carbon polyhedron is hollow with dense CNTs rooted on the surface (TEM images are not shown here). During the heat treatment, the $\mathrm{H}_{2}$ atmosphere plays a critical role in the growth of the carbon nanotubes. Metallic Co nanoparticles are quickly formed in the presence of the $\mathrm{H}_{2}$ atmosphere, followed by the catalytic growth of carbon nanotubes (Figure 4). In this process, ZIF-67 particles serve not only as the $\mathrm{C}$ and $\mathrm{N}$ source for growth of carbon nanotubes, but also as the self-template for the framework morphology (Xia et al., 2016). As reported in literature, only solid porous carbon polyhedrons without carbon nanotubes containing cobalt were formatted in argon

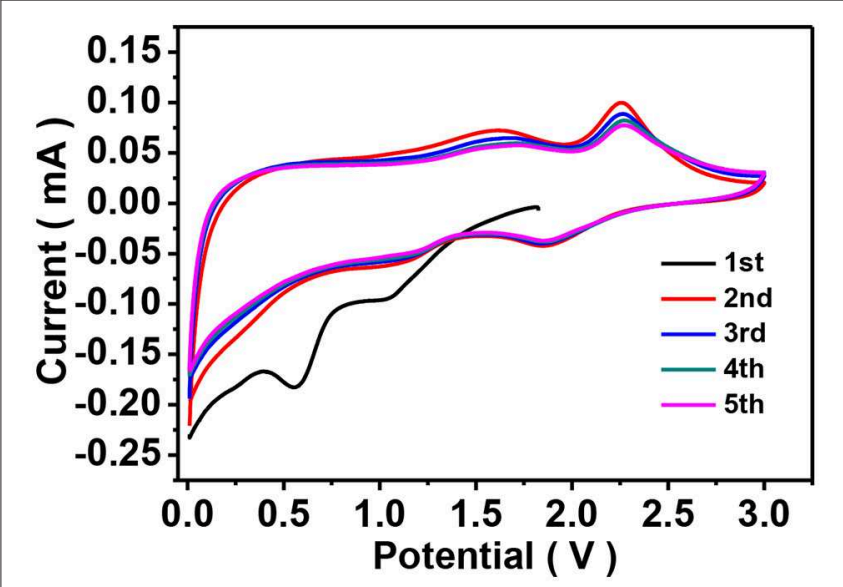

FIGURE 5 | CV curves of the ZIF-67-C@MoS 2 composite as LIB anodes in the voltage range of $0.01-3.0 \mathrm{~V}$ at a scan rate of $0.1 \mathrm{mV} \mathrm{s}^{-1}$.

or nitrogen atmosphere (Lu et al., 2018). The SEM images of ZIF-67-C@MoS 2 composite are shown in Figures 3a-c. $\mathrm{MoS}_{2}$ nanosheets were uniformly coated on the surface of ZIF-67$\mathrm{C}$, and Figure $3 \mathrm{c}$ clearly shows the ultrathin $\mathrm{MoS}_{2}$ flakes on the ZIF-67-C@MoS 2 surface. Elemental mappings of ZIF-67$\mathrm{C} @ \mathrm{MoS}_{2}$ in Figures 3d-h shows the presence and uniform distribution of $\mathrm{C}, \mathrm{O}, \mathrm{Mo}$, and $\mathrm{S}$ elements in the composite, indicating the uniform distribution of $\mathrm{MoS}_{2}$ nanosheets on the surface of ZIF-67-C.

To explore the phase composition, Figure 4 shows the XRD patterns of the three materials. In the XRD pattern of ZIF-67, the peaks at about $2 \theta$ located at 10.3, 14.7, 16.4, 18.9, 21.1, 24.6, 25.6, 26.7, 29.6 corresponds to the (002), (022), (013), (222), (114), 
A

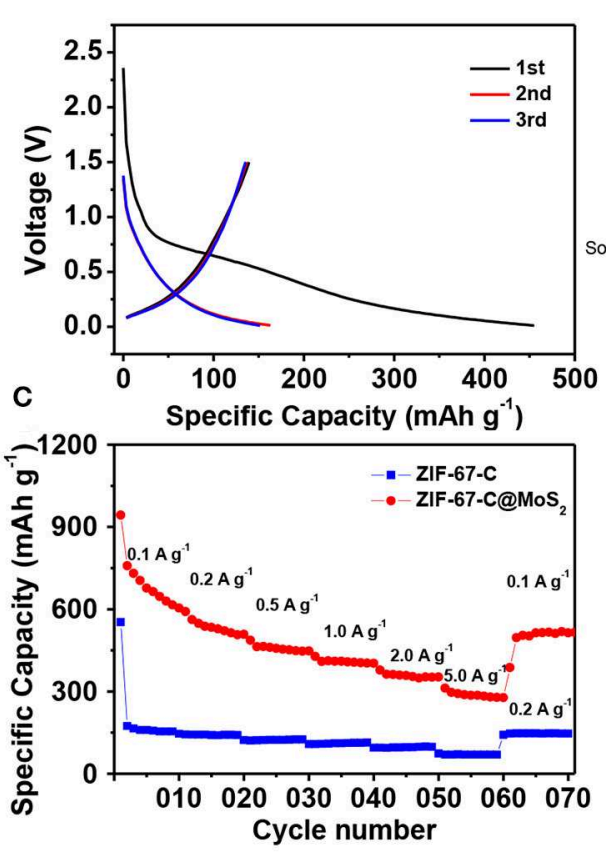

B

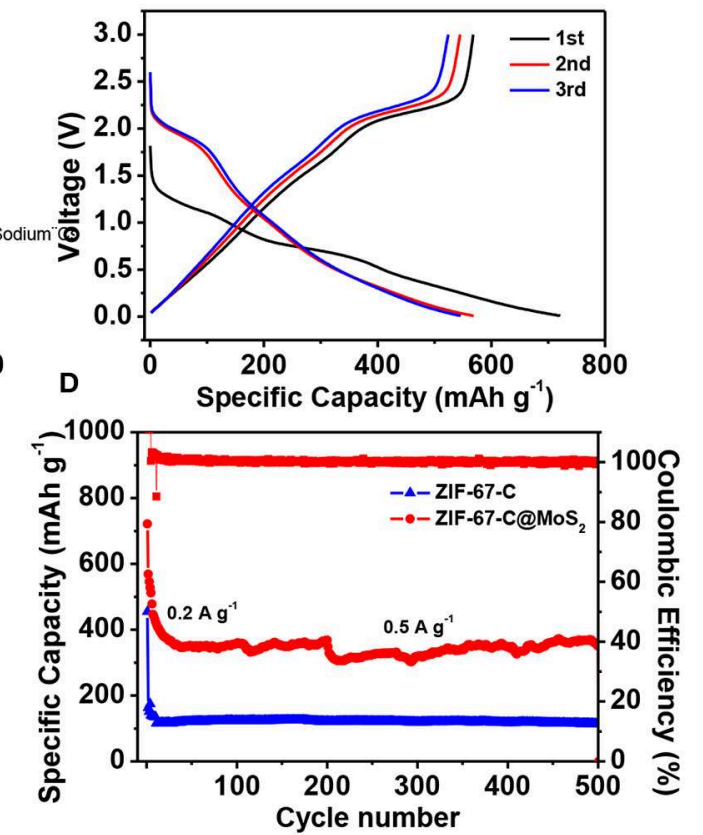

FIGURE 6 | Electrochemical performance of ZIF-67-C and ZIF-67-C@MoS 2 in LIBs. (A) Charge/discharge curves of ZIF-67-C at 0.2 A g ${ }^{-1}$ in the 1st, 2nd and 3rd cycles in a voltage range of 0.01-1.5 V. (B) Charge/discharge curves of ZIF-67-C@MoS 2 at $0.1 \mathrm{~A} \mathrm{~g}^{-1}$ in the 1st, 2nd, and 3rd cycles between 0.01 and $3 \mathrm{~V}$. (C) Rate capability at different rates. (D) Cycling performance of two electrodes at a current density of 0.2 and $0.5 \mathrm{~A} \mathrm{~g}^{-1}$.

(233), (224), (134), and (044) plane, respectively (Gross et al., 2012; Lin and Chang, 2015), indicating that the prepared ZIF67 sample is a pure phase. For the XRD pattern of ZIF-67-C, the peak at about $25.2^{\circ}$ corresponds to the carbon peak, and the other peaks at about $44.3^{\circ}, 51.6^{\circ}, 75.9^{\circ}$ can be attributed to metallic Co. The XRD pattern of ZIF-67-C@ $\mathrm{MoS}_{2}$, the diffraction peaks at $2 \theta$ of 33.2, 39.5, and 59.4 corresponded to the (100), (103), and (110) planes of the $\mathrm{MoS}_{2}$ (Zhang et al., 2018a).

Lithium storage properties of the as-prepared ZIF-67-C and ZIF-67-C@MoS 2 materials were investigated by galvanostatic discharge-charge and CV tests using CR2032 coin cells. To evaluate the electrochemical activity of the ZIF-67-C@MoS composite, $\mathrm{CV}$ measurements were carried out at a scan rate of $0.1 \mathrm{mV} \mathrm{s}^{-1}$. As shown in Figure 5, during the first cathodic scan, the peak located at $1.04 \mathrm{~V}$ can be attributed to the $\mathrm{Li}^{+}$ intercalation into $\mathrm{MoS}_{2}$ to form $\mathrm{Li}_{\mathrm{x}} \mathrm{MoS}_{2}$ accompanied by a phase change of $\mathrm{MoS}_{2}$ from $2 \mathrm{H}$ to $1 \mathrm{~T}$. And the peak at $0.56 \mathrm{~V}$ corresponds to the conversion reaction of $\mathrm{Li}_{\mathrm{X}} \mathrm{MoS}_{2}$ into metallic Mo particles and $\mathrm{Li}_{2} \mathrm{~S}$ (Teng et al., 2016). The broad peak in the range of $0.5-0.8 \mathrm{~V}$ can be attributed to the formation of SEI layers. In the subsequent anodic scans, two peaks at 1.60 and $2.25 \mathrm{~V}$ are observed. The weak peak at $1.60 \mathrm{~V}$ can be assigned to a partial oxidation of Mo to form $\mathrm{MoS}_{2}$. The sharp oxidation peak at $2.25 \mathrm{~V}$ indicates delithiation of $\mathrm{Li}_{2} \mathrm{~S}$ to sulfur. In the following scan, the cathodic peak shifts to $1.87 \mathrm{~V}$, corresponding to the a conversion of $\mathrm{S}$ to polysulfide and the formation of $\mathrm{Li}_{2} \mathrm{~S}$. Other peak at $1.20 \mathrm{~V}$ can be ascribed to lithiation with metal Mo nanoparticles.
Figure 6A shows the discharge/charge curves of the ZIF-67-C sample cycling in the potential range of $0.01-1.5 \mathrm{~V}$ at a constant current density of $0.2 \mathrm{~A} \mathrm{~g}^{-1}$. The initial discharge capacity is $454.9 \mathrm{mAh} \mathrm{g}^{-1}$, the initial charge capacity is $139.2 \mathrm{mAh} \mathrm{g}^{-1}$, corresponding a low Coulombic efficiency (CE) of $30.6 \%$, which can be contributed to the formation of SEI film in the first discharge process. The reversible capacity in the second and third cycle is 163 and $151.5 \mathrm{mAh} \mathrm{g}^{-1}$, respectively. The electrode shows one sloping voltage plateau at about $0.31 \mathrm{~V}$ (vs. $\mathrm{Li}^{+} / \mathrm{Li}$ ) (Jiao et al., 2017), typical charge-discharge curves for carbon material. As shown in Figure 6B, the ZIF-67-C@ $\mathrm{MoS}_{2}$ composite delivers the highest specific discharge capacity of $721.2 \mathrm{mAh} \mathrm{g}^{-1}$ at $0.1 \mathrm{~A}$ $\mathrm{g}^{-1}$, and a high efficiency of $78.7 \%$. Furthermore, the electrode exhibits a higher reversible capacities of 568.5 and $545.9 \mathrm{mAh} \mathrm{g}^{-1}$ in the second and third cycles at the same current density.

Figure 6C compares the rate performance of the two materials at different current rates. It is clear that the reversible capacity of ZIF-67-C@ $\mathrm{MoS}_{2}$ composite is much better than that of ZIF67-C at various current density. At a low rate of $0.1 \mathrm{~A} \mathrm{~g}^{-1}$, the ZIF-67-C@MoS 2 delivered a discharge capacity of $758.7 \mathrm{mAh}$ $\mathrm{g}^{-1}$. Furthermore, the ZIF-67-C@MoS 2 sample could deliver discharge capacities of 561.7, 462.8, 409.3, and $362.8 \mathrm{mAh} \mathrm{g}^{-1}$ at the current rates of $0.2,0.5,1$, and $2 \mathrm{~A} \mathrm{~g}^{-1}$, implying the excellent rate capability of the ZIF-67-C@MoS 2 electrode. Even at high rate of $5 \mathrm{~A} \mathrm{~g}^{-1}$, a high discharge capacity of $296.7 \mathrm{mAh} \mathrm{g}^{-1}$ can still be obtained. When the current density returns to $0.1 \mathrm{~A} \mathrm{~g}^{-1}$, the discharge capacity is $504.7 \mathrm{mAh} \mathrm{g}^{-1}$, corresponding to $89.8 \%$ retention of the discharge capacity. 
The cycling performance of the two materials is also examined. As shown in Figure 6D, all electrodes showed very stable capacity retention at a current density of 0.2 and $0.5 \mathrm{~A}$ $\mathrm{g}^{-1}$. For ZIF-67-C@MoS 2 , the first five cycles are activated at a low current density of $0.1 \mathrm{~A} \mathrm{~g}^{-1}$, then cycling at $0.2 \mathrm{~A} \mathrm{~g}^{-1}$ to 200 cycles, and further tested at $0.5 \mathrm{~A} \mathrm{~g}^{-1}$ for another 300 cycles. The ZIF-67-C@MoS 2 exhibits a discharge capacity of $350.4 \mathrm{mAh} \mathrm{g}^{-1}$ after 500 cycles. In contrast, the capacity of ZIF-67-C electrode

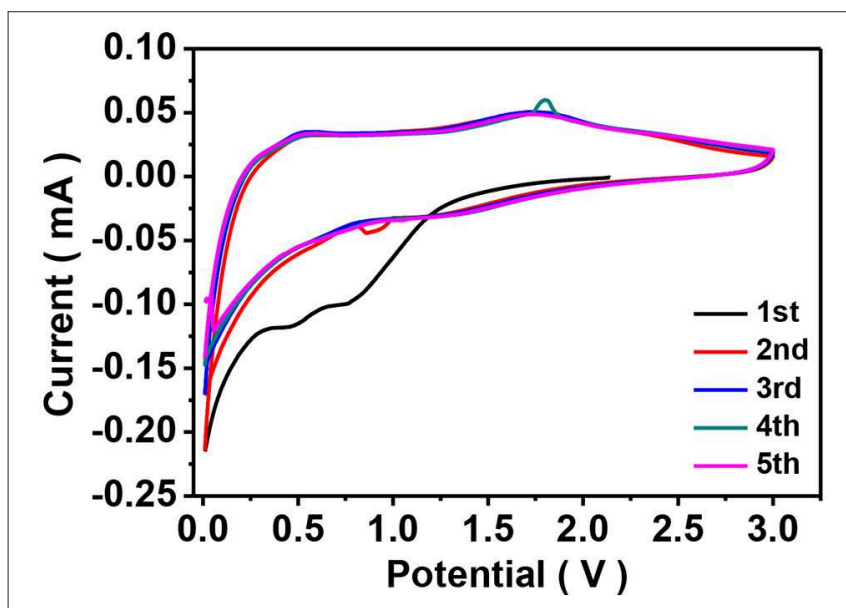

FIGURE 7 | CV curves of the ZIF-67-C@MoS 2 composite as PIB anodes at the scan rate of $0.1 \mathrm{mV} \mathrm{s}^{-1}$. falls to lower than $114.2 \mathrm{mAh} \mathrm{g}^{-1}$ at $0.5 \mathrm{~A} \mathrm{~g}^{-1}$ (activating at $0.2 \mathrm{~A} \mathrm{~g}^{-1}$ for 10 cycles). The enhanced cycling stability of the ZIF-67-C@MoS 2 samples results from the $\mathrm{MoS}_{2}$ coating, and synergistic effect of merits of ZIF-67-C, which could efficiently improve electronic conductivity and increased specific capacity.

As mentioned above, the ZIF-67-C@MoS 2 favored fast lithium ions diffusion and improved reaction kinetics with more active sites for the $\mathrm{Li}^{+}$insertion/extraction due to the large surface area, effectively increasing the specific capacity of composite. However, it is urgent to find a new generation of energy storage devices due to the limited content of lithium resources in earth. The content of potassium in the earth's crust is $1.5 \%$, which is much higher than that of lithium (0.0017\%) (Zhang et al., 2019a). Moreover, the standard reduction potential of $\mathrm{K} / \mathrm{K}^{+}(-2.92 \mathrm{~V}$ vs. SHE) is close to that of $\mathrm{Li} / \mathrm{Li}^{+}(-3.04 \mathrm{~V}$ vs. SHE) and lower than that of $\mathrm{Na} / \mathrm{Na}^{+}(-2.71 \mathrm{~V}$ vs. SHE). Thus, potassium ion batteries (PIBs) have potential application prospect for high energy devices (Chen et al., 2018b; Zhang et al., 2019b).

We furthermore investigated the potassium storage properties of ZIF-67-C@MoS 2 as new anodes. The CV curves of ZIF67-C@ $\mathrm{MoS}_{2}$ composite were firstly tested in the voltage range of $0.01-3.0 \mathrm{~V}$ at a scan rate of $0.1 \mathrm{mV} \mathrm{s}^{-1}$ (Figure 7). During the first cathodic sweep, an obvious reduction peak in the range of $1.2-0.5 \mathrm{~V}$ can be clearly seen, which corresponds to the potassiation of $\mathrm{MoS}_{2}$ and $\mathrm{K}$ to form the $\mathrm{K}_{\mathrm{X}} \mathrm{MoS}_{2}$ (Ren et al., 2017), the irreversible decomposition of the electrolyte and formation of the SEI layers. The peak in $0.01-0.5 \mathrm{~V}$ can be attributed to the further reaction of $\mathrm{K}_{\mathrm{x}} \mathrm{MoS}_{2}$ and $\mathrm{K}^{+}$to

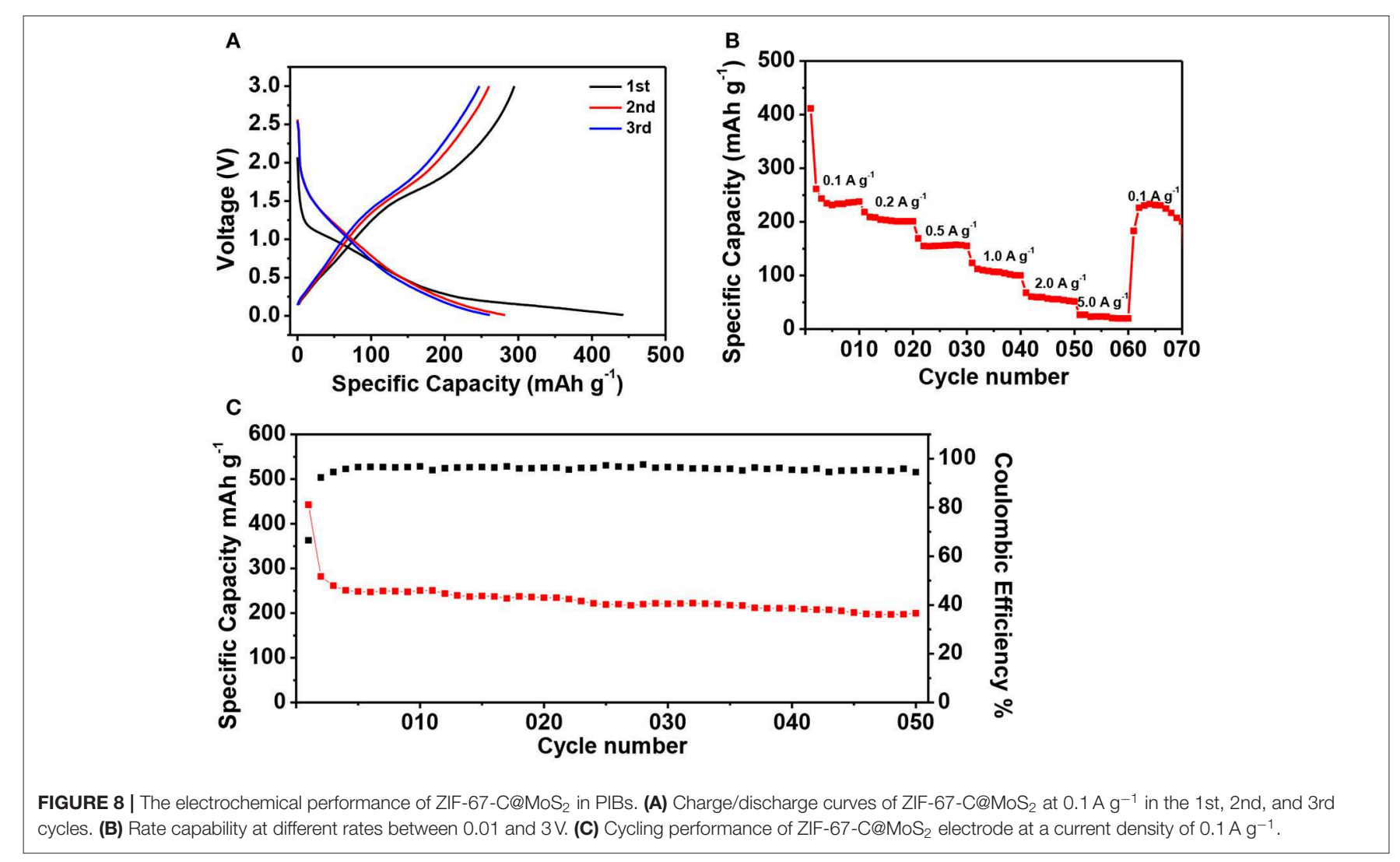


form Mo particles and $\mathrm{K}_{2} \mathrm{~S}$. During the subsequent anodic scan, the peak at $1.75 \mathrm{~V}$ can be attributed to the deintercalation of K-ion and the oxidation of Mo to $\mathrm{MoS}_{2}$ (Chen et al., 2018d). Figure 8 shows the potassium ion storage performance of the ZIF-67-C@MoS 2 electrode with metal potassium as counter electrode. The galvanostatic charge/discharge curves are tested in the potential range of $0.01-3 \mathrm{~V}$ at $0.1 \mathrm{~A} \mathrm{~g}^{-1}$ in Figure 8A. The initial discharge and charge capacity is 442.7 and $294.6 \mathrm{mAh} \mathrm{g}^{-1}$, corresponding to an initial CE of $66.5 \%$. The large capacity loss in the first cycle is mainly due to the decomposition of the electrolyte and the formation of the SEI layer. The discharge capacity is 282.0 and $261.2 \mathrm{mAh}$ $\mathrm{g}^{-1}$ in the second and third cycles, respectively. The electrode shows one voltage plateau at about $0.75 \mathrm{~V}$ (vs. $\mathrm{K}^{+} / \mathrm{K}$ ). The rate capacity of the ZIF-67-C@MoS 2 electrode is illustrated at various current densities from $0.1,0.2,0.5,1,2$, and $5 \mathrm{~A} \mathrm{~g}^{-1}$, as shown in Figure 8B. They deliver high reversible discharge capacities of 261.5, 208.8, 155.1, 112.3, 60.5, and $26.4 \mathrm{mAh}$ $\mathrm{g}^{-1}$, respectively. Moreover, when back to $0.1 \mathrm{~A} \mathrm{~g}^{-1}$, the ZIF67-C@MoS 2 electrode still shows a capacity of $226.3 \mathrm{mAh}$ $\mathrm{g}^{-1}$, demonstrating an excellent rate performance. The cycling performance of the ZIF-67-C@MoS 2 is also examined as shown in Figure 8C, the initial discharge capacity is $442.7 \mathrm{mAh} \mathrm{g}^{-1}$, and delivers a high discharge capacity of $199.8 \mathrm{mAh} \mathrm{g}^{-1}$ after 50 cycles at a current density of $0.1 \mathrm{~A} \mathrm{~g}^{-1}$ with the $\mathrm{CE}$ of close to $100 \%$. All in all, the composite exhibits superior rate performance and cycling stability in both lithium-ion and potassium-ion batteries, showing promising potential for large energy storage application.

\section{CONCLUSIONS}

In summary, ultrathin $\mathrm{MoS}_{2}$ rooted on cobalt nanoparticlescontaining porous carbon polyhedral with dense carbon nanotubes has been synthesized by using ZIF-67 as self-template and precursor. During the pyrolysis process, ZIF-67 not only provides the $\mathrm{C}$ and $\mathrm{N}$ source for growth of $\mathrm{N}$-doped carbon nanotubes arrays catalyzed by the in-situ formed metallic Co nanoparticles but also serves as the template for the formation of the hollow polyhedron. The obtained ZIF-67$\mathrm{C} @ \mathrm{MoS}_{2}$ composite exhibits improved specific capacity and

\section{REFERENCES}

Basdogan, Y., and Keskin, S. (2015). Simulation and modelling of MOFs for hydrogen storage. CrystEngComm 17, 261-275. doi: 10.1039/C4CE01711K

Chen, K., Sun, Z., Fang, R., Shi, Y., Cheng, H.-M., and Li, F. (2018a). Metalorganic frameworks (MOFs)-derived nitrogen-doped porous carbon anchored on graphene with multifunctional effects for lithium-sulfur batteries. $A d v$. Funct. Mater. 28:1707592. doi: 10.1002/adfm.201870274

Chen, L.-F., Feng, Y., Liang, H.-W., Wu, Z.-Y., and Yu, S.-H. (2017a). Macroscopic-scale three-dimensional carbon nanofiber architectures for electrochemical energy storage devices. Adv. Energy Mater. 7:1700826. doi: 10.1002/aenm.201700826

Chen, L.-F., Lu, Y., Yu, L., and Lou, X. W. (2017b). Designed formation of hollow particle-based nitrogen-doped carbon nanofibers for high-performance supercapacitors. Energ. Environ. Sci. 10, 1777-1783. doi: 10.1039/C7EE00488E excellent rate capabilities compared to the pristine ZIF-67-C electrode for lithium ion batteries and potassium ion batteries. In particular, the ZIF-67-C@MoS shows the superior lithium storage properties, delivering high reversible capacities of 568.5 $\mathrm{mAh} \mathrm{g}^{-1}$ at $0.2 \mathrm{~A} \mathrm{~g}^{-1}$, superior rate capability (high discharge capacity of $291.9 \mathrm{mAh} \mathrm{g}^{-1}$ at $5 \mathrm{~A} \mathrm{~g}^{-1}$ ), and excellent cycling stability for over 500 cycles. The enhanced electrochemical performance of the ZIF-67-C@ $\mathrm{MoS}_{2}$ material could be attributed to the beneficial effects of highly-conductive carbon network with nitrogen doped CNTs and cobalt particle, large specific surface and robust hollow structure. The results here provide a facile strategy for large-scale synthesis of high performance anode materials through low cost perspective, and new insights for designing MOF-derived functional materials with unique structures for energy storage application.

\section{DATA AVAILABILITY STATEMENT}

All datasets generated for this study are included in the article.

\section{AUTHOR CONTRIBUTIONS}

$\mathrm{BR}, \mathrm{LC}$, and PN conceived the project and wrote the manuscript. $\mathrm{BR}$ carried out the materials synthesis. BR, JL, and HW conducted the materials characterization and the electrochemical evaluation of lithium ion batteries. JL, YG, and LL conducted the tests of potassium ion batteries and the related data processing. All authors discussed the results, read, and commented on the manuscript.

\section{FUNDING}

This work was supported by the National Key R\&D Program of China (No. SQ2017YFGH001474), National Natural Science Foundation of China (Nos. 51802111, 51778268), Research Program on Science and Technology from the Education Department of Jilin Province (No. JJKH20190997KJ), Funding of Research Program of Jilin Normal University, JLNU Innovation Program for Graduate Education (No. 201919), and Science and Technology Development Project of Siping City (No. 2017053). 
Chen, Z., Peng, Y., Liu, F., Le, Z., Zhu, J., Shen, G., et al. (2015). Hierarchical nanostructured $\mathrm{WO}_{3}$ with biomimetic proton channels and mixed ionicelectronic conductivity for electrochemical energy storage. Nano Lett. 15, 6802-6808. doi: 10.1021/acs.nanolett.5b02642

Chen, Z., Yin, D., and Zhang, M. (2018d). Sandwich-like $\mathrm{MoS}_{2} @ \mathrm{SnO}_{2} @ \mathrm{C}$ with high capacity and stability for sodium/potassium ion batteries. Small 14:1703818. doi: 10.1002/smll.201870074

Choi, J. W., and Aurbach, D. (2016). Promise and reality of post-lithiumion batteries with high energy densities. Nat. Rev. Mater. 1:16013. doi: $10.1038 /$ natrevmats.2016.13

Davies, D. M., Verde, M. G., Mnyshenko, O., Chen, Y. R., Rajeev, R., Meng, Y. S., et al. (2019). Combined economic and technological evaluation of battery energy storage for grid applications. Nat. Energy 4, 42-50. doi: 10.1038/s41560-018-0290-1

Ding, M., Chen, G., Xu, W., Jia, C., and Luo, H. (2019). Bio-inspired synthesis of nanomaterials and smart structures for electrochemical energy storage and conversion. Nano Mater. Sci. doi: 10.1016/j.nanoms.2019.09.011. [Epub ahead of print].

Dunn, B., Kamath, H., and Tarascon, J.-M. (2011). Electrical energy storage for the grid: a battery of choices. Science 334, 928-935. doi: 10.1126/science.12 12741

Gadipelli, S., Li, Z., Lu, Y., Li, J., Guo, J., Skipper, N. L., et al. (2019). Size-related electrochemical performance in active carbon nanostructures: a MOFs-derived carbons case study. Adv. Sci. 0:1901517. doi: 10.1002/advs.201901517

Griffith, K. J., Wiaderek, K. M., Cibin, G., Marbella, L. E., and Grey, C. P. (2018). Niobium tungsten oxides for high-rate lithium-ion energy storage. Nature 559, 556-563. doi: 10.1038/s41586-018-0347-0

Gross, A. F., Sherman, E., and Vajo, J. J. (2012). Aqueous room temperature synthesis of cobalt and zinc sodalite zeolitic imidizolate frameworks. Dalton Trans. 41, 5458-5460. doi: 10.1039/c2dt30174a

Jagadeesh, R. V., Murugesan, K., Alshammari, A. S., Neumann, H., Pohl, M. M., Radnik, J., et al. (2017). MOF-derived cobalt nanoparticles catalyze a general synthesis of amines. Science 358, 326-332. doi: 10.1126/science.aan6245

Jiang, J., Zhang, Y., An, Y., Wu, L., Zhu, Q., Dou, H., et al. (2019). Engineering ultrathin $\mathrm{MoS}_{2}$ nanosheets anchored on N-doped carbon microspheres with pseudocapacitive properties for high-performance lithium-ion capacitors. Small Methods 3:1900081. doi: 10.1002/smtd.201900081

Jiang, Z., Li, Z., Qin, Z., Sun, H., Jiao, X., and Chen, D. (2013). LDH nanocages synthesized with MOF templates and their high performance as supercapacitors. Nanoscale 5, 11770-11775. doi: 10.1039/c3nr03829g

Jiao, Y., Mukhopadhyay, A., Yang, L., and Zhu, H. (2017). Ion transport nanotube assembled with vertically aligned metallic $\mathrm{MoS}_{2}$ for high rate lithium-ion batteries. Adv. Energy Mater. 8:1702779. doi: 10.1002/aenm.201702779

Kim, J., Yeo, S., Jeon, J.-D., and Kwak, S.-Y. (2015). Enhancement of hydrogen storage capacity and hydrostability of metal-organic frameworks (MOFs) with surface-loaded platinum nanoparticles and carbon black. Microporous Mesoporous Mater. 202, 8-15. doi: 10.1016/j.micromeso.2014.09.025

Kovalenko, I., Zdyrko, B., Magasinski, A., Hertzberg, B., Milicev, Z., Burtovyy, R., et al. (2011). A major constituent of brown algae for use in high-capacity Li-Ion batteries. Science 334, 75-79. doi: 10.1126/science.1209150

Le, Z., Liu, F., Nie, P., Li, X., Liu, X., Bian, Z., et al. (2017). Pseudocapacitive sodium storage in mesoporous single-crystal-like $\mathrm{TiO}_{2}$-graphene nanocomposite enables high-performance sodium-ion capacitors. ACS Nano 11, 2952-2960. doi: 10.1021/acsnano.6b08332

Li, Y., Yan, K., Lee, H.-W., Lu, Z., Liu, N., and Cui, Y. (2016). Growth of conformal graphene cages on micrometre-sized silicon particles as stable battery anodes. Nat. Energy 1:15029. doi: 10.1038/nenergy.2016.17

Lin, K. Y., and Chang, H. A. (2015). Ultra-high adsorption capacity of zeolitic imidazole framework-67 (ZIF-67) for removal of malachite green from water. Chemosphere 139, 624-631. doi: 10.1016/j.chemosphere.2015.01.041

Liu, X., Huang, W., Wang, D., Tian, J., and Shan, Z. (2017). A nitrogen-doped 3D hierarchical carbon/sulfur composite for advanced lithium sulfur batteries. J. Power Sources 355, 211-218. doi: 10.1016/j.jpowsour.2017.04.067

Liu, X., Li, X., Li, H., and Wu, H. B. (2018). Recent progress of hybrid solid-state electrolytes for lithium batteries. Chem. Eur. J. 24, 18293-18306. doi: 10.1002/chem.201803616

Lu, H., Zhang, C., Zhang, Y., Huang, Y., Liu, M., and Liu, T. (2018). Simultaneous growth of carbon nanotubes on inner/outer surfaces of porous polyhedra: advanced sulfur hosts for lithium-sulfur batteries. Nano Res. 11, 6155-6166. doi: 10.1007/s12274-018-2130-9

Mínguez Espallargas, G., and Coronado, E. (2018). Magnetic functionalities in MOFs: from the framework to the pore. Chem. Soc. Rev. 47, 533-557. doi: 10.1039/C7CS00653E

Nie, P., Le, Z., Chen, G., Liu, D., Liu, X., Wu, H. B., et al. (2018). Graphene caging silicon particles for high-performance lithium-ion batteries. Small 14:1800635. doi: 10.1002/smll.201800635

Nie, P., Liu, X., Fu, R., Wu, Y., Jiang, J., Dou, H., et al. (2017a). Mesoporous silicon anodes by using polybenzimidazole derived pyrrolic $\mathrm{N}$-enriched carbon toward high-energy Li-ion batteries. ACS Energy Lett. 2, 1279-1287. doi: 10.1021/acsenergylett.7b00286

Nie, P., Shen, L., Luo, H., Ding, B., Xu, G., Wang, J., et al. (2014). Prussian blue analogues: a new class of anode materials for lithium ion batteries. J. Mater. Chem. A 2, 5852-5857. doi: 10.1039/C4TA00062E

Nie, P., Shen, L., Pang, G., Zhu, Y., Xu, G., Qing, Y., et al. (2015). Flexible metalorganic frameworks as superior cathodes for rechargeable sodium-ion batteries. J. Mater. Chem. A 3, 16590-16597. doi: 10.1039/C5TA03197D

Nie, P., Yuan, J., Wang, J., Le, Z., Xu, G., Hao, L., et al. (2017b). Prussian blue analogue with fast kinetics through electronic coupling for sodium ion batteries. ACS Appl. Mater. Interfaces 9, 20306-20312. doi: 10.1021/acsami.7b05178

Novoselov, K. S., Geim, A. K., Morozov, S. V., Jiang, D., Zhang, Y., Dubonos, S. V., et al. (2004). Electric field effect in atomically thin carbon films. Science 306, 666-669. doi: 10.1126/science.1102896

Oveisi, M., Asli, M. A., and Mahmoodi, N. M. (2018). MIL-Ti metal-organic frameworks (MOFs) nanomaterials as superior adsorbents: synthesis and ultrasound-aided dye adsorption from multicomponent wastewater systems. J. Hazard Mater. 347, 123-140. doi: 10.1016/j.jhazmat.2017.12.057

Peng, Y., Le, Z., Wen, M., Zhang, D., Chen, Z., Bin Wu, H., et al. (2017). Mesoporous single-crystal-like $\mathrm{TiO}_{2}$ mesocages threaded with carbon nanotubes for high-performance electrochemical energy storage. Nano Energy 35, 44-51. doi: 10.1016/j.nanoen.2017.03.003

Ren, X., Zhao, Q., McCulloch, W. D., and Wu, Y. (2017). $\mathrm{MoS}_{2}$ as a longlife host material for potassium ion intercalation. Nano Res. 10, 1313-1321. doi: 10.1007/s12274-016-1419-9

Stephenson, T., Li, Z., Olsen, B., and Mitlin, D. (2014). Lithium ion battery applications of molybdenum disulfide $\left(\mathrm{MoS}_{2}\right)$ nanocomposites. Energy Environ. Sci. 7, 209-231. doi: 10.1039/C3EE42591F

Sun, F., Liu, X., Wu, H. B., Wang, L., Gao, J., Li, H., et al. (2018). In situ high-level nitrogen doping into carbon nanospheres and boosting of capacitive charge storage in both anode and cathode for a high-energy $4.5 \mathrm{~V}$ full-carbon lithiumion capacitor. Nano Lett. 18, 3368-3376. doi: 10.1021/acs.nanolett.8b00134

Sun, F., Wang, K., Wang, L., Pei, T., Gao, J., Zhao, G., et al. (2019). Hierarchical porous carbon sheets with compressed framework and optimized pore configuration for high-rate and long-term sodium and lithium ions storage. Carbon 155, 166-175. doi: 10.1016/j.carbon.2019.08.051

Teng, Y., Zhao, H., Zhang, Z., Li, Z., Xia, Q., Zhang, Y., et al. (2016). MoS nanosheets vertically grown on graphene sheets for lithium-ion battery anodes. ACS Nano 10, 8526-8535. doi: 10.1021/acsnano.6b03683

Tian, Y., Liu, X., Cao, X., Zhang, D., Xiao, S., Li, X., et al. (2019). Microwave-assisted synthesis of $1 \mathrm{~T} \mathrm{MoS}_{2} / \mathrm{Cu}$ nanowires with enhanced capacity and stability as anode for LIBs. Chem. Eng. J. 374, 429-436. doi: 10.1016/j.cej.2019.05.174

Wang, C., Wu, H., Chen, Z., McDowell, M. T., Cui, Y., and Bao, Z. (2013). Self-healing chemistry enables the stable operation of silicon microparticle anodes for high-energy lithium-ion batteries. Nat. Chem. 5:1042. doi: $10.1038 /$ nchem.1802

Wang, J., Tang, J., Ding, B., Chang, Z., Hao, X., Takei, T., et al. (2018) Self-template-directed metal-organic frameworks network and the derived honeycomb-like carbon flakes via confinement pyrolysis. Small 14:1704461. doi: 10.1002/smll.201704461

Wang, R., Jin, D., Zhang, Y., Wang, S., Lang, J., Yan, X., et al. (2017) Engineering metal organic framework derived 3D nanostructures for high performance hybrid supercapacitors. J. Mater. Chem. A 5, 292-302. doi: 10.1039/C6TA09143A

Wu, H. B., and Lou, X. W. D. (2017). Metal-organic frameworks and their derived materials for electrochemical energy storage and conversion: 
promises and challenges. Sci. Adv. 3:eaap9252. doi: 10.1126/sciadv. aap9252

Xia, B. Y., Yan, Y., Li, N., Wu, H. B., Lou, X. W., and Wang, X. (2016). A metalorganic framework-derived bifunctional oxygen electrocatalyst. Nat. Energy 1:15006. doi: 10.1038/nenergy.2015.6

Xiao, J., Choi, D., Cosimbescu, L., Koech, P., Liu, J., and Lemmon, J. P. (2010). Exfoliated $\mathrm{MoS}_{2}$ nanocomposite as an anode material for lithium ion batteries. Chem. Mater. 22, 4522-4524. doi: 10.1021/cm101254j

Xie, K., Yuan, K., Li, X., Lu, W., Shen, C., Liang, C., et al. (2017). Superior potassium ion storage via vertical $\mathrm{MoS}_{2}$ "Nano-Rose" with expanded interlayers on graphene. Small 13:1701471. doi: 10.1002/smll.201701471

Xu, G., Nie, P., Dou, H., Ding, B., Li, L., and Zhang, X. (2017). Exploring metal organic frameworks for energy storage in batteries and supercapacitors. Mater. Today 20, 191-209. doi: 10.1016/j.mattod.2016.10.003

Xu, X., Zhao, R., Ai, W., Chen, B., Du, H., Wu, L., et al. (2018). Controllable design of $\mathrm{MoS}_{2}$ nanosheets anchored on nitrogen-doped graphene: toward fast sodium storage by tunable pseudocapacitance. Adv. Mater. 30:1800658. doi: 10.1002/adma.201800658

Xue, D. X., Cairns, A. J., Belmabkhout, Y., Wojtas, L., Liu, Y., Alkordi, M. H., et al. (2013). Tunable rare-earth fcu-MOFs: a platform for systematic enhancement of $\mathrm{CO}_{2}$ adsorption energetics and uptake. J. Am. Chem. Soc. 135, 7660-7667. doi: $10.1021 /$ ja401429x

Yunhong, P., Xiyi, L., Qibin, X., Junliang, W., Yingwei, L., Jiang, X., et al. (2017). Adsorptive and photocatalytic removal of persistent organic pollutants (POPs) in water by metal-organic frameworks (MOFs). Chem. Eng. J. 337, 351-371. doi: 10.1016/j.cej.2017.12.092

Zhang, L., Ji, X., Ren, X., Ma, Y., Shi, X., Tian, Z., et al. (2018a). Electrochemical ammonia synthesis via nitrogen reduction reaction on a $\mathrm{MoS}_{2}$ catalyst: theoretical and experimental studies. Adv. Mater. 30:1800191. doi: 10.1002/adma.201800191
Zhang, P., Wang, F., Yu, M., Zhuang, X., and Feng, X. (2018b). Twodimensional materials for miniaturized energy storage devices: from individual devices to smart integrated systems. Chem. Soc. Rev. 47, 7426-7451. doi: 10.1039/C8CS00561C

Zhang, W., Liu, Y., and Guo, Z. (2019a). Approaching high-performance potassium-ion batteries via advanced design strategies and engineering. Sci. Adv. 5:eaav7412. doi: 10.1126/sciadv.aav7412

Zhang, X., Yang, Y., Song, L., Wang, Y., He, C., Wang, Z., et al. (2018c). High and stable catalytic activity of $\mathrm{Ag} / \mathrm{Fe}_{2} \mathrm{O}_{3}$ catalysts derived from MOFs for CO oxidation. Mol. Catal. 447, 80-89. doi: 10.1016/j.mcat.2018.01.007

Zhang, Z., Jia, B., Liu, L., Zhao, Y., Wu, H., Qin, M., et al. (2019b). Hollow multihole carbon bowls: a stress-release structure design for high-stability and high-volumetric-capacity potassium-ion batteries. ACS Nano 13, 11363-11371. doi: 10.1021/acsnano.9b04728

Zhu, C., Mu, X., van Aken, P. A., Yu, Y., and Maier, J. (2014). Single-layered ultrasmall nanoplates of $\mathrm{MoS}_{2}$ embedded in carbon nanofibers with excellent electrochemical performance for lithium and sodium storage. Angew. Chem. Int. Ed. 53, 2152-2156. doi: 10.1002/anie.201308354

Conflict of Interest: The authors declare that the research was conducted in the absence of any commercial or financial relationships that could be construed as a potential conflict of interest.

Copyright (c) 2019 Rui, Li, Chang, Wang, Lin, Guo and Nie. This is an open-access article distributed under the terms of the Creative Commons Attribution License (CC BY). The use, distribution or reproduction in other forums is permitted, provided the original author(s) and the copyright owner(s) are credited and that the original publication in this journal is cited, in accordance with accepted academic practice. No use, distribution or reproduction is permitted which does not comply with these terms. 